\title{
Genetic gain of pure line selection and combined crossbred purebred selection with constrained inbreeding
}

\author{
P. Bijma ${ }^{1}$, J. A. Woolliams ${ }^{2}$ and J. A. M. van Arendonk ${ }^{1}$ \\ ${ }^{1}$ Animal Breeding and Genetics Group, Wageningen Institute of Animal Sciences, Wageningen University, \\ 6700 AH Wageningen, The Netherlands \\ ${ }^{2}$ Roslin Institute (Edinburgh), Roslin, Midlothian EH25 9PS, UK
}

\begin{abstract}
Using deterministic methods, rates of genetic gain $(\Delta G)$ and inbreeding $(\Delta F)$ were compared between pure line selection (PLS) and combined crossbred purebred selection (CCPS), for the sire line of a three-way crossbreeding scheme. Purebred performance and crossbred performance were treated as genetically correlated traits assuming the infinitesimal model. Breeding schemes were compared at a fixed total number of purebred selection candidates, i.e. including crossbred information did not affect the size of the purebred nucleus. Selection was by truncation on estimated breeding values for crossbred performance. Rates of genetic gain were predicted using a pseudo-BLUP selection index. Rates of inbreeding were predicted using recently developed methods based on long-term genetic contributions. Results showed that changing from PLS to CCPS may increase $\Delta F$ by a factor of 2.14. In particular with high heritabilities and low purebred-crossbred genetic correlations, CCPS requires a larger number of parents than PLS, to avoid excessive $\triangle F$. The superiority of CCPS over PLS was judged by comparing $\triangle G$ from both selection strategies at the same $\triangle F$. At the same $\triangle F, C C P S$ was superior to PLS and the superiority of CCPS was only moderately reduced compared with the situation without a restriction on $\Delta F$. This paper shows that the longterm genetic contribution theory can be used to balance $\Delta F$ and $\Delta G$ in animal breeding schemes within very limited computing time.
\end{abstract}

Keywords: crossbreeding, effective population size, genetic gain, inbreeding, selection.

\section{Introduction}

In crossbreeding programmes, the primary aim is to increase the performance of the crossbred animals. For this reason, the breeding goal should be defined on the crossbred level, though some weight may be given to purebred performance in particular cases (Jiang and Groen, 1999). Several studies have shown that selection response in crossbred performance can be increased by including both purebred and crossbred information in the selection criterion (Wei and van der Werf, 1994; Baumung et al., 1997; Bijma and van Arendonk, 1998; Uimari and Gibson, 1998). Purebred performance and crossbred performance can be treated as genetically different traits, and selection may be based on an estimated breeding value for crossbred performance (EBV), which uses both purebred and crossbred information (Wei and van der Werf, 1994; Bijma and van Arendonk, 1998). Such a selection strategy is referred to as combined crossbred purebred selection (CCPS), whereas selection based solely on information from the pure line is referred to as pure line selection (PLS). None of the studies mentioned above, however, has considered the effect of CCPS on the rate of inbreeding. As will be argued next, the use of CCPS may substantially increase the rate of inbreeding compared with PLS.

In a CCPS programme, an important information source is the phenotypic information on crossbred half sibs of the selection candidate (Wei and van der Werf, 1994). For example, in a three-way crossbreeding system, sires from the sire line may simultaneously be mated to dams of the sire line and to F1 dams from the multiplier level of the dam line. In that case, sires from the sire line produce two types of offspring; purebred offspring within the sire line, which are the selection candidates for the next generation, and commercial crossbred offspring which provide information on crossbred performance. When estimating breeding values for 
the purebred selection candidates, the information on their crossbred half-sibs can be included in the EBV, which results in a higher accuracy of selection. Wei and van der Werf (1993) and Spilke et al. (1998) show how mixed model equations can be set up to estimate genetic parameters and breeding values with CCPS.

When the breeding goal is crossbred performance and the genetic correlation between purebred and crossbred performance $\left(r_{p c}\right)$ is low, the information coming from crossbred half sibs will dominate the EBVs of selection candidates. As a consequence, there will be a high intraclass correlation between EBVs of full- and half-sibs present among the purebred selection candidates. Depending on the value of $r_{p c}$ therefore, selection in a CCPS programme may tend to between family selection, resulting in increased rates of inbreeding. To maintain genetic variation and to limit inbreeding depression in the pure line, rates of inbreeding have to be restricted in animal breeding programmes. The relevant question, therefore, is whether the superiority of CCPS schemes over PLS schemes can be sustained when rates of inbreeding are restricted. This question has not been addressed so far.

The aim of this paper is to compare CCPS schemes to PLS schemes while restricting the rate of inbreeding. First, we will compare the intraclass correlation between EBVs of sibs for PLS and CCPS schemes. Subsequently, rates of genetic gain and rates of inbreeding will be compared between CCPS and PLS schemes which have the same size of pure line. Finally, we will maximize genetic gain from PLS and CCPS schemes by optimizing the number of selected parents while restricting the rate of inbreeding. The superiority of CCPS schemes over PLS schemes will be judged by comparing rates of gain from both selection strategies at the same rate of inbreeding.

\section{Methods}

Traits

Purebred and crossbred performance will be treated as two different traits having a genetic correlation of $r_{p c}$. It is assumed that both traits are determined by the infinitesimal model, that phenotypic variance equals one for both traits and that heritability is equal for both traits.

\section{Population structure}

The population structure was the same as the structure considered by Bijma and van Arendonk (1998), and will be described briefly here. The sire line of a three-way crossbreeding system in pigs was modelled. Mating structure was hierarchical and generations were discrete. Sires from the sire line were mated to $n_{p d}$ purebred dams from the sire line to produce $n_{p o}$ purebred offspring per dam $\left(1 / 2 n_{p o}\right.$ of each sex). At the same time, the same sires were also mated to F1-dams from the multiplier level of the dam line, to produce $n_{c b}$ crossbred fattening pigs per sire. Each purebred selection candidate, therefore, had $n_{c b}$ crossbred half sibs providing crossbred information. Purebred performance was measured on animals within the sire line, and crossbred performance was measured on fattening pigs. Information on purebred and crossbred individuals was assumed to be available at the same time. The traits were measured on both sexes and each sire had the same number of offspring tested. Each generation, $n_{s}$ males and $n_{s} n_{p d}$ females were selected out of the $1 / 2 N=1 / 2 n_{s} n_{p d} n_{p o}$ purebred selection candidates of each sex, to become parents of the next generation. Selection was by truncation on EBV for crossbred performance, without a restriction on the number of parents contributed by a single family.

\section{Rates of gain}

Throughout this paper, rate of genetic gain in crossbred performance with PLS is denoted as $\Delta G_{p l s}$ and rate of genetic gain in crossbred performance with CCPS is denoted as $\Delta G_{c c p s}$. Rates of genetic gain in crossbred performance were predicted using the pseudo-BLUP selection index of Bijma and van Arendonk (1998). This index is an extension of the single trait pseudo-BLUP index of Wray and Hill (1989) to CCPS, and combines phenotypic information of the selection candidate and its fulland half-sibs together with EBVs of parents into a pseudo-BLUP EBV for crossbred performance of the selection candidate. The pseudo-BLUP index predicts response in crossbred performance for a situation with multi-trait animal model BLUP evaluation, including all purebred, crossbred and pedigree information, and where selection is by truncation on the EBV for crossbred performance. Detailed equations of the index are described by Bijma and van Arendonk (1998).

A minor modification was made to the index of Bijma and van Arendonk (1998). Bijma and van Arendonk (1998) explicitly modelled the F1-dams, but here we will assume that EBVs of F1-dams are not available, which is likely to be the situation in practice. Consequently, the F1-dams were omitted from the index, and the total number of information sources equalled 9, whereas the index of Bijma and van Arendonk (1998) contained 10 information sources.

Selection intensities accounted for finite population size and intraclass correlations between EBVs of relatives, and were calculated using the method of 
Meuwissen (1991). Selection and mating were iterated until Bulmer's (1971) equilibrium parameters were reached, using the equations described by Bijma and van Arendonk (1998). Rates of genetic gain were predicted for the equilibrium situation.

\section{Rates of inbreeding}

Rates of inbreeding $(\Delta \mathrm{F})$ were predicted deterministically using the long-term genetic contribution theory (Woolliams et al., 1999; Woolliams and Bijma, 2000). The application of this theory to CCPS is a direct analogy of the method to predict $\Delta \mathrm{F}$ for single trait BLUP selection as described by Bijma and Woolliams (2000). Here we will only outline the main steps involved in predicting $\Delta \mathrm{F}$, detailed equations can be found in Bijma and Woolliams (2000).

The procedure for predicting $\Delta \mathrm{F}$ consisted of the following steps. First, Bulmer's (1971) equilibrium genetic parameters were obtained by iterating on the pseudo-BLUP index of Bijma and van Arendonk (1998). Subsequently, the intraclass correlation between EBVs of full- and half-sibs were calculated from the selection index equations, following the approach described in appendix A of Bijma and Woolliams (2000). Finally, rates of inbreeding were predicted using equations 1 through 13 and appendix B of Bijma and Woolliams (2000). In equations 2 and 3 of Bijma and Woolliams (2000), the single trait breeding value was replaced by the breeding value for crossbred performance. For single trait BLUP selection, an example of computation is given in appendix B of Bijma and Woolliams (2000). In the present paper, the accuracy of the predicted rate of inbreeding will be evaluated by means of stochastic simulation for a number of CCPS schemes.

\section{Optimization of breeding schemes}

Breeding schemes were optimized by maximizing genetic gain while restricting the rate of inbreeding. Optimization was performed at different fixed values of the mating ratio $\left(n_{p d}\right)$ and of the total number of selection candidates $(N)$. The optimization variable was the number of sires, i.e. the number of sires was increased until the inbreeding constraint was achieved. The number of dams and the number of offspring per dam was a result of the number of sires and the mating ratio, i.e. the number of dams was $n_{s} n_{p d}$ and the number of offspring per dam was $n_{p o}=N /\left(n_{s} n_{p d}\right)$. The number of offspring per dam was allowed to be a non-integer value, because the primary purpose of this paper is to examine trends, and not to present values for specific actual breeding schemes.

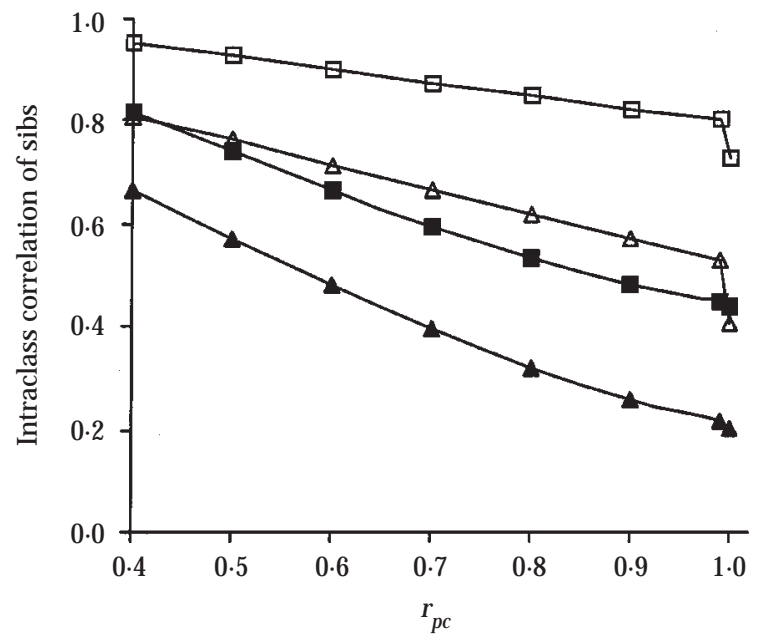

Figure 1 Intraclass correlations between EBVs of full- and half-sibs with CCPS, as a function of $r_{p c}$, for $n_{s}=20, n_{p d}=$ 3, $n_{p o}=4$ and $n_{c h}=60 . \square \square-$ full-sibs, $h^{2}=0.15$; $\triangle$ half-sibs, $h^{2}=0.15 ; \square$ full-sibs, $h^{2}=0.6$; half-sibs, $h^{2}=0.6$; The symbols at $k_{p c}=1$ represent intraclass correlations for PLS.

The optimization is in one dimension, i.e. the only variable is the number of sires, and from $n_{s} \geq 12$ and higher, $\Delta F$ and $\Delta G$ are continuously decreasing functions of $n_{s}$, which makes it easy to identify the optimum scheme. With increasing $n_{s}$, the optimum scheme, i.e. the optimum $n_{s}$, is the first scheme where $\Delta F$ is smaller than or equal to the constraint, and the maximum possible $\Delta G$ given the constraint on $\Delta F$ is the rate of gain from this scheme. The optimality of CCPS $v$. PLS was judged by comparing $\Delta G_{p l s}$ and $\Delta G_{c c p s}$ for the optimum schemes of both selection strategies, at the same rate of inbreeding and for the same total number of selection candidates.

\section{Results}

Intraclass correlation between EBVs of sibs

Figure 1 shows the intraclass correlation between EBVs of full- and half-sibs with CCPS, as a function of the purebred-crossbred genetic correlation $\left(r_{p c}\right)$ and for two different heritabilities, $h^{2}=0.15$ or $h^{2}=0.6$. (Note that purebred and crossbred performance were assumed to have the same heritability. ) With $r_{p c}=1$, purebred and crossbred performance are the same trait, so for this situation, CCPS corresponds to single trait selection. With $h^{2}=0 \cdot 15$ and $r_{p c}=1$, intraclass correlations are high, which shows that with low heritability single trait BLUP selection puts substantial emphasis on family information. With $h^{2}=0.6$ and $r_{p c}=1$, intraclass correlations are substantially lower, because 
BLUP-EBV are largely determined by individual performance when heritability is high. With decreasing $r_{p c}$ the intraclass correlation increases rapidly for $h^{2}=0 \cdot 6$, because emphasis shifts from the purebred individual performance to family information for crossbred performance. For $h^{2}=0 \cdot 15$, the increase is smaller because family information already receives substantial emphasis for $r_{p c}=1$. With PLS the intraclass correlation is slightly smaller than with CCPS and $r_{p c}=1$, because with CCPS there are an additional 60 half-sibs included in the EBV. In conclusion, intraclass correlations increase with decreasing $r_{p c}$ and the increase is largest for high heritabilities.

\section{Rates of inbreeding and gain}

Figure 2 shows the rate of inbreeding with CCPS as a function of $r_{p c}$ for different heritabilities and for two different selection intensities, $n_{p o}=4$ or 8 . To evaluate the accuracy of the deterministic predictions (lines in Figure 2), Figure 2 also shows $\Delta F$ estimated from stochastic simulation (symbols in Figure 2), which reveals that predictions and simulations are in close agreement. A discussion on the origin of prediction errors is in Bijma and Woolliams (2000).

Figure 2 shows that $\Delta F$ increases with decreasing $r_{p c}$ and that the relative increase is largest with high

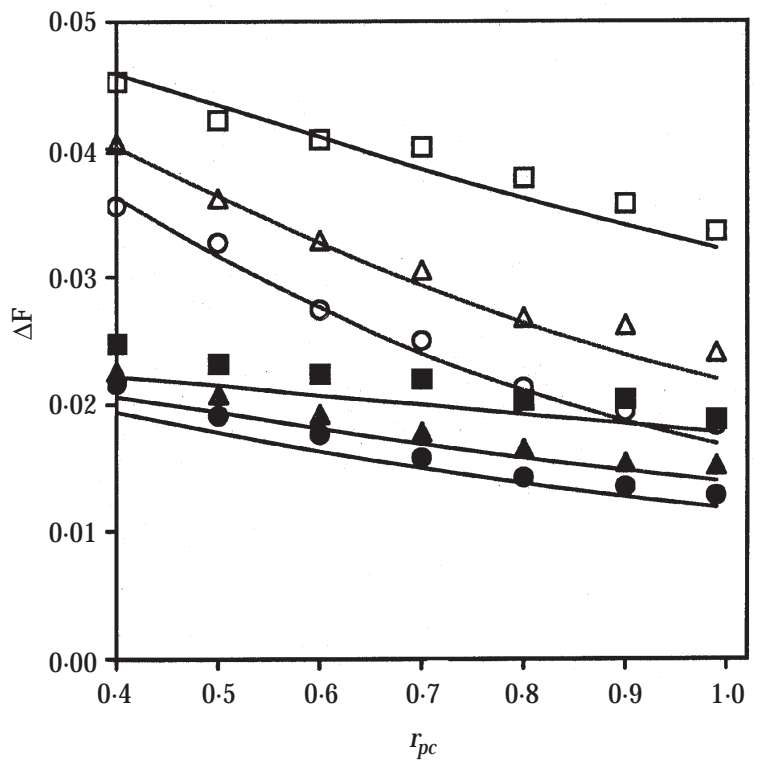

Figure 2 Rates of inbreeding $(\Delta F)$ with CCPS as a function of $n_{p c}$, for $n_{s}=20, n_{p d}=3$ and $n_{c b}=60$. $\square-n_{p o}=8, h^{2}=$ $0.15 ; \triangle n_{p o}=8, h^{2}=0.4 ; \longrightarrow n_{p o}=8, h^{2}=0.6$; $\longrightarrow n_{p o}=4, h^{2}=0.15 ; \longrightarrow n_{p o}=4, h^{2}=0.4 ; \longrightarrow n_{p o}$ $=4, h^{2}=0 \cdot 6$. Lines represent predictions, symbols represent results from simulation. heritabilities and high selection intensities. For example, with $n_{p o}=8$ and $h^{2}=0 \cdot 6$, the rate of inbreeding increased by a factor of 2.14 when $r_{p c}$ decreased from 1 to $0 \cdot 4$; whereas with $h^{2}=0 \cdot 15$, the rate of inbreeding increased only by a factor of 1.42 . The large increase of $\Delta F$ for high heritabilities agrees with the trend in the intraclass correlations observed in Figure 1.

Figure 2 also shows a rapid increase of $\Delta F$ when selection intensity increases. For example, with $h^{2}=0.15$ and $r_{p c}=1, \Delta F$ increased by a factor of 1.81 when the number of purebred offspring per dam was doubled from 4 to 8 ; and for $r_{p c}=0 \cdot 4, \Delta F$ increased by an even greater factor 2.06. This shows that, with BLUP selection, doubling the number of selection candidates while keeping the number of parents constant may double the rate of inbreeding. Obviously, the rate of inbreeding with PLS (results not shown) is independent of $r_{p c}$ and was slightly lower than $\Delta F$ for CCPS with $r_{p c}=1$. In conclusion, Figure 2 shows that, when keeping the number of parents constant, changing from PLS to CCPS may substantially increase $\Delta F$, in particular for small $r_{p c}$, high $h^{2}$ and high selection intensity.

Figure 3 shows $\Delta G_{p l s}$ and $\Delta G_{c c p s}$ as a function of $r_{p c}$ and for different heritabilities. With PLS, genetic gain

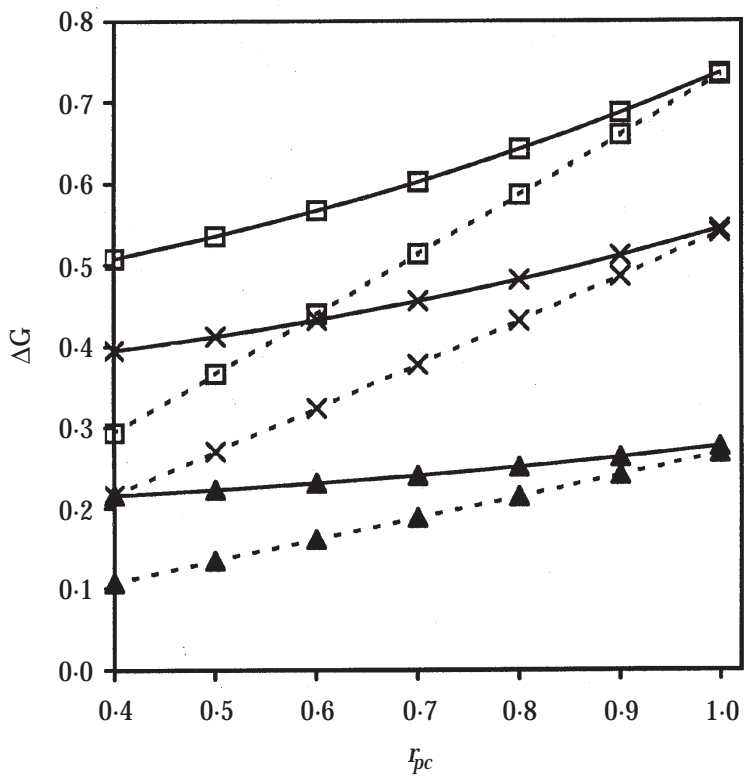

Figure 3 Rates of genetic gain in crossbred performance $(\Delta G)$ as a function of $r_{p c}$ for $n_{s}=20, n_{p d}=3, n_{p o}=8$ and $n_{c b}=$ 60. For CCPS: $\square \square-h^{2}=0.6 ; \longrightarrow h^{2}=0.4 ; \longrightarrow h^{2}$ $=0 \cdot 15$. For PLS: $--\square---h^{2}=0 \cdot 6 ;--\nless-\cdots h^{2}=0 \cdot 4 ;--\mathbf{A}--h^{2}=$ $0 \cdot 15$. 
in crossbred performance is equal to the product of $\Delta G_{\text {purebred }}$ and $r_{p c}$, and, therefore, $\Delta G_{p l s}$ increases linearly with $r_{p c}$. As expected from previous studies (e.g. Wei and van der Werf, 1994), CCPS gives more genetic gain than PLS and the superiority of CCPS over PLS increases with decreasing $r_{p c}$. The relative superiority of CCPS over PLS is largest for low heritabilities. For example, with $r_{p c}=0.4$ and $h^{2}=0 \cdot 15$, using CCPS instead of PLS increased genetic gain by a factor 2.02, whereas for $h^{2}=0 \cdot 6$, genetic gain increased only by a factor of 1.73 .

Combining Figures 2 and 3 shows, that for high heritabilities together with high selection intensities, $\Delta \mathrm{F}$ increases more rapidly than $\Delta \mathrm{G}$ when changing from PLS to CCPS. For example, for $h^{2}=0 \cdot 6, r_{p c}=0.4$ and $n_{p o}=8$, changing from PLS to CCPS increased $\Delta \mathrm{G}$ by a factor of $1 \cdot 73$ and $\Delta \mathrm{F}$ by a factor of $2 \cdot 14$.

\section{PLS v. CCPS with constrained inbreeding}

To make inferences on the superiority of CCPS over PLS when the rate of inbreeding is restricted, the ratio $\Delta G_{c c p s} / \Delta G_{p l s}$ was calculated for schemes where genetic gain was maximized while restricting $\Delta F$. Figure 4 shows $\Delta G_{c c p s} / \Delta G_{p l s}$ for optimum schemes with a fixed total number of 768 pure line selection candiates, where $\Delta F$ was restricted to be smaller than $1 \%$. To validate the deterministic optimization, a limited number of schemes was optimized using stochastic simulation (results not shown), which

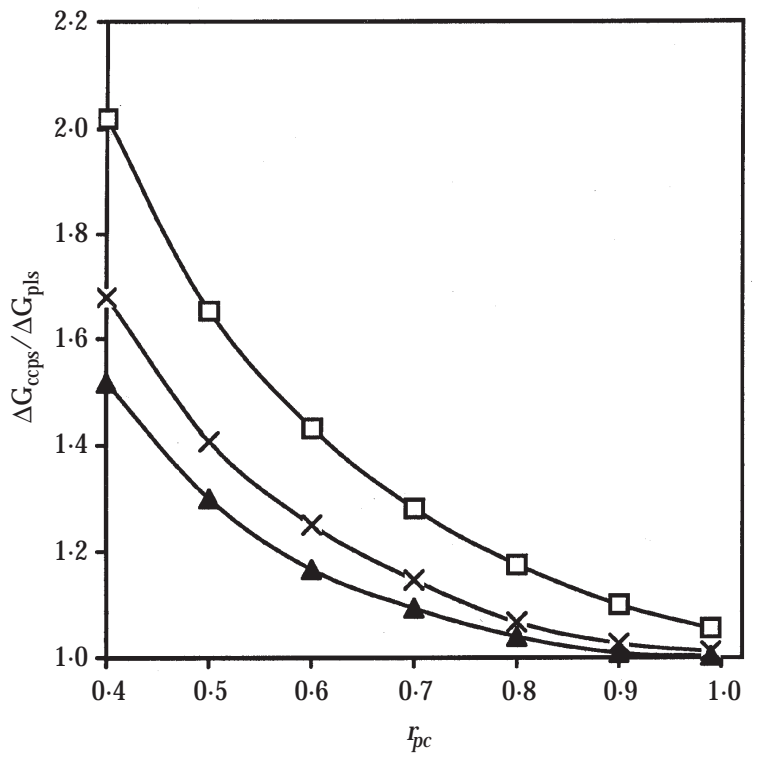

Figure 4 Ratio of genetic gain with CCPS over genetic gain with PLS $\left(\Delta G_{c c p s} / \Delta G_{p l s}\right)$ as a function of $r_{p c}$, for optimum schemes with $\Delta F<1 \%$ and with $N=768, n_{p d}=5$ and $n_{c b}=$ 60. $\square \square-h^{2}=0.15 ; \longrightarrow h^{2}=0.4 ; \longrightarrow h^{2}=0.6$.

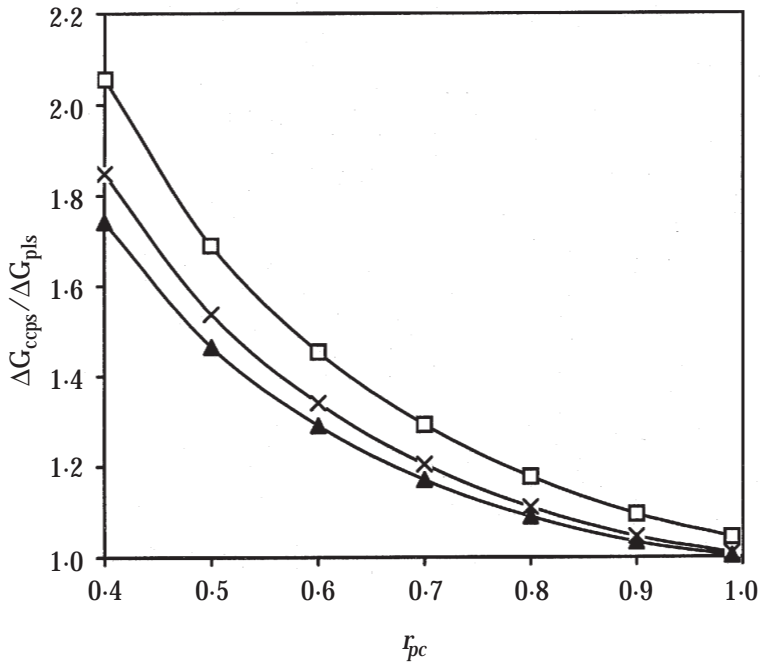

Figure 5 Ratio of genetic gain with CCPS over genetic gain with PLS $\left(\Delta G_{c c p s} / \Delta G_{p l s}\right)$ as a function of $r_{p c}$, without a restriction on $\Delta F$, for $n_{s}=30, n_{p d}=5, n_{p o}=6$, and $n_{c b}=60$.

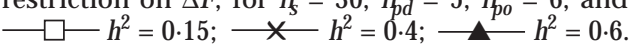

revealed close agreement between predictions and simulations. Results in Figure 4 show that, with a restriction on the rate of inbreeding, CCPS remains superior over PLS, i.e. the ratio $\Delta G_{c c p s} / \Delta G_{p l s}$ exceeds one. The superiority of CCPS over PLS increases with decreasing $r_{p c}$ and with decreasing $h^{2}$. The ratio $\Delta G_{c c p s} / \Delta G_{p l s}$ was also evaluated for other values of $N$ and $d$, but the relation was almost identical, which indicates that with constrained inbreeding, the superiority of CCPS over PLS is determined almost entirely by $r_{p c}$ and $h^{2}$. Results for other values of $N$ and $d$, therefore, are not shown.

Figure 5 shows the superiority of CCPS over PLS without a restriction on the rate of inbreeding, for a scheme with $n_{s}=30, n_{p d}=5$ and $n_{p o}=6$. For $h^{2}=0.15$, the ratio $\Delta G_{c c p s} / \Delta G_{p l s}$ is very similar to the situation where $\Delta F$ is restricted, e.g. with $r_{p c}=0.4$ and no restriction on $\Delta F$ the ratio was 2.06 (Figure 5), whereas with $\Delta F<1 \%$ the ratio was 2.02 . For low heritabilities, therefore, restricting $\Delta F$ hardly affects the superiority of CCPS over PLS. For higher heritabilities the superiority of CCPS over PLS decreased when $\Delta F$ was restricted, e.g. with $h^{2}=0 \cdot 6$, $r_{p c}=0.4$ and no restriction on $\Delta F$ the ratio was 1.74 (Figure 5), whereas with $\Delta F<1 \%$ the ratio decreased to 1.51. Compared with the large increase of $\Delta F$ when changing from CCPS to PLS (Figure 2), the reduction of $\Delta G_{c c p s} / \Delta G_{p l s}$ when $\Delta F$ is restricted is strikingly small. 
Table 1 shows the design of optimum breeding schemes, i.e. the optimum number of selected sires, for different constraints on the rate of inbreeding. With PLS, the optimum number of sires is always lower than with CCPS and decreases when $h^{2}$ increases. With CCPS the optimum number of sires decreases with increasing $h^{2}$ and $r_{p c}$. With decreasing $r_{p c}$ the required number of sires increased rapidly for $h^{2}=0 \cdot 6$, whereas for $h^{2}=0 \cdot 15$ there is only a small increase. This result agrees with the observation that the increase of intraclass correlations is largest for high heritabilities (Figure 1). Results in Table 1 indicate that, with a constraint on $\Delta F$, the difference between the optimum number of sires with PLS and CCPS increases with heritability. For low heritabilities, this difference is relatively small because single trait BLUP selection already puts substantial emphasis on family information.

The comparison of CCPS $v$. PLS at fixed rates of inbreeding involves a trade off between accuracy and intensity of selection. Table 1 shows that with CCPS the constraint on $\Delta F$ is achieved by selecting more parents, which is at the expense of selection intensity. On the other hand, CCPS benefits from a higher accuracy of selection due to the use of crossbred

Table 1 Optimum number of selected sires for CCPS and PLS schemes with constrained inbreeding $(\Delta F) \dagger$

\begin{tabular}{|c|c|c|c|c|}
\hline$h^{2}$ & $r_{p c}$ & $\Delta F<2 \%$ & $\Delta F<1 \%$ & $\Delta F<0.5 \%$ \\
\hline \multirow[t]{8}{*}{$0 \cdot 15$} & 0.4 & 32 & 44 & 61 \\
\hline & 0.5 & 31 & 44 & 60 \\
\hline & 0.6 & 30 & 43 & 59 \\
\hline & 0.7 & 29 & 42 & 58 \\
\hline & 0.8 & 28 & 41 & 57 \\
\hline & 0.9 & 27 & 40 & 56 \\
\hline & 0.99 & 26 & 39 & 55 \\
\hline & PLS & 26 & 38 & 53 \\
\hline \multirow[t]{8}{*}{$0 \cdot 4$} & $0.4^{\top}$ & 30 & 42 & 59 \\
\hline & 0.5 & 28 & 41 & 58 \\
\hline & 0.6 & 27 & 39 & 56 \\
\hline & 0.7 & 25 & 37 & 54 \\
\hline & 0.8 & 24 & 36 & 53 \\
\hline & 0.9 & 22 & 34 & 51 \\
\hline & 0.99 & 21 & 32 & 49 \\
\hline & PLS $\ddagger$ & 21 & 32 & 49 \\
\hline \multirow[t]{8}{*}{0.6} & 0.4 & 28 & 41 & 58 \\
\hline & 0.5 & 27 & 39 & 56 \\
\hline & 0.6 & 24 & 37 & 54 \\
\hline & 0.7 & 22 & 34 & 51 \\
\hline & $0 \cdot 8$ & 20 & 32 & 49 \\
\hline & 0.9 & 19 & 30 & 46 \\
\hline & 0.99 & 17 & 28 & 44 \\
\hline & PLS $\ddagger$ & 17 & 28 & 44 \\
\hline
\end{tabular}

+ For $N=768, n_{p d}=5$ and $n_{c b}=60, h^{2}=$ heritability, $r_{p c}=$ purebred-crossbred genetic correlation.

$\ddagger$ With PLS the optimum design is independent of $r_{p c}$.

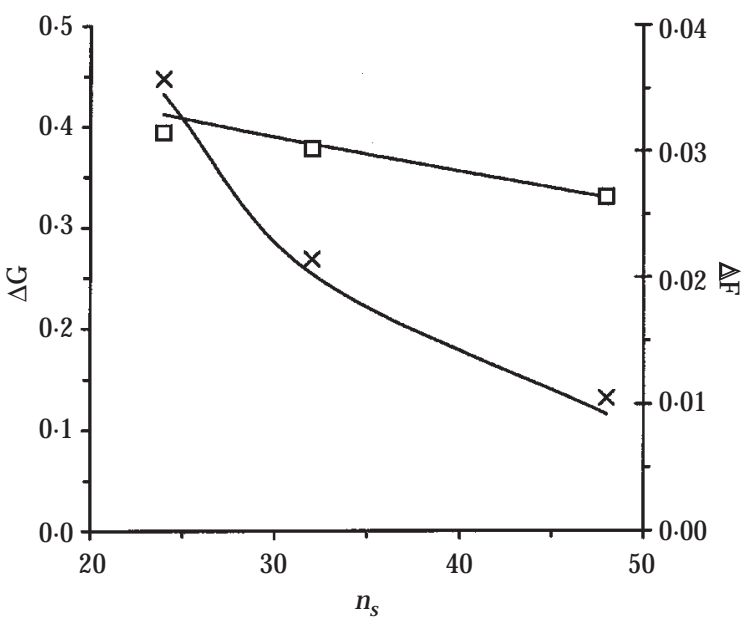

Figure 6 Relation of $\Delta F$ and $\Delta G$ with the number of selected sires $n_{s}$ for a fixed test capacity of $N=768$, with $n_{p d}$ $=4, h^{2}=0.4, r_{p c}=0.4$ and $n_{c b}=60 ; \square \square-\Delta G ; \stackrel{\square}{\longrightarrow} \Delta F$. Lines represent predictions, symbols represent results from simulation.

information. The superiority of CCPS vs. PLS at fixed rates of inbreeding (Figure 4) indicates that the increase of gain due to increased accuracy exceeds the decrease of gain due to decreased selection intensity.

Given the large differences in $\Delta F$ between CCPS and PLS (Figure 2), the difference between the optimum number of sires for both selection strategies (Table 1) is surprisingly small. This indicates that a small increase in the number of sires is sufficient to substantially reduce $\Delta F$. Figure 6 shows the relation of $\Delta F$ and $\Delta G$ with the number of selected sires, for $h^{2}=0.4, r_{p c}=0.4$ and $N=768$, which shows that $\Delta F$ drops substantially when the number of sires increases, whereas $\Delta G$ decreases only moderately. There are two distinct mechanisms causing the sharp decrease of $\Delta F$. First, in the absence of selection, $\Delta F$ approximately halves when the number of parents is doubled (Wright, 1969). Second, with fixed $N$, selection intensity decreases when the number of parents increases, which further reduces $\Delta F$ (see appendix B of Bijma and Woolliams (2000)). For the scheme in Figure 6, both mechanisms contribute approximately equally to the reduction of $\Delta F$. The different relation of $\Delta F$ and $\Delta G$ with the number of sires also explains why the superiority of CCPS over PLS, i.e $\Delta G_{c c p s} / \Delta G_{p l s}$, reduces only moderately when $\Delta F$ is restricted (Figure $4 v$. Figure 5). 


\section{Discussion}

In this paper, we have compared CCPS to PLS with particular emphasis on the rate of inbreeding. When keeping the number of parents constant, changing from PLS to CCPS may substantially increase $\Delta F$. In particular with high $h^{2}$ and low $r_{p c}$ CCPS requires a larger number of parents than PLS, to avoid excessive rates of inbreeding. When compared at the same rate of inbreeding, CCPS was superior to PLS and the superiority of CCPS was only moderately reduced compared with a situation without a restriction on $\Delta F$.

Until recently, balancing rates of gain and inbreeding in animal breeding programmes required computationally demanding stochastic simulation, which seriously restricted the number of alternative schemes involved in the optimization. This paper shows that the use of the long-term genetic contribution theory for predicting $\Delta F$ (Woolliams et al., 1999; Bijma and Woolliams, 2000; Woolliams and Bijma, 2000) enables the optimization of rates of gain and inbreeding of breeding schemes, within very limited computing time with no restriction on the number of alternative schemes involved. For example, the results in Figure 4, which involved the evaluation of approx. 750 alternative breeding schemes, were generated within nine wall clock seconds.

Following the approach of Wei and van der Werf (1994), we have treated purebred performance and crossbred performance as correlated traits, assuming the additive infinitesimal model. Other studies have used finite locus models with several degrees of dominance, and generally with equal effects for all loci (Baumung et al., 1997; Uimari and Gibson, 1998). Neither of these models is fully realistic, i.e. the true number of genes must be finite, but on the other hand we have little knowledge of the number of genes, their effects and the interactions between genes.

Prediction of short-term selection response by means of selection index theory does not require the infinitesimal assumptions, providing gene effects are not very large and gene frequency of favourable alleles is near 0.5 (Hill, 1998). When phenotypes and breeding values follow a multivariate normal distribution, the regression of breeding values on phenotypes, i.e. the selection index, is unbiased. With multivariate normality, therefore, selection index theory exactly predicts response to a single cycle of selection. Although selection may induce deviations from normality, Turelli and Barton (1994) show that the prediction of genetic gain by regression on phenotypes is robust against deviations from normality, even with strong truncation selection. Therefore, prediction of response by means of selection index theory is expected to be adequate in the short term.

In the long-term, selection response depends on the nature of the genetic variation, i.e. the number of genes, their effects etc. However, we have little knowledge of those parameters, and it will be difficult to convince practitioners to sacrifice shortterm response (i.e. by not using CCPS) when it remains doubtful whether doing so will indeed yield more long-term response. The most practical approach, therefore, seems to be the use of CCPS while restricting the rate of inbreeding, so as to maximize short-term response and simultaneously avoid rapid erosion of the genetic variation. To account for changing genetic parameters due to changing gene frequency, genetic parameters should frequently be re-estimated so that optimality of the CCPS EBV is guaranteed.

In this study, breeding schemes were optimized given a fixed total number of purebred selection candidates, whereas the number of recorded crossbred individuals varied between schemes. In other studies, crossbred individuals were tested at the expense of purebred individuals, thus reducing the number of selection candidates (Wei and van der Werf, 1994). Whether testing crossbred individuals is at the expense of purebred individuals depends on whether testing of crossbred individuals is part of the nucleus-breeding programme, or, alternatively, information from the commercial population is collected, e.g. at the slaughterhouses. Collecting information at the slaughterhouse has the advantage that one can directly measure the traits that determine the carcass price, e.g. carcass grade and lean \%, instead of an ultrasonic measurement of backfat, which may increase accuracy of selection. Additionally, measuring crossbred performance on individuals of the commercial population has the advantage that $\mathrm{G} \times \mathrm{E}$ interaction is accounted for. When crossbred information is collected outside the nucleus, PLS and CCPS are not compared at the same total amount of resources, so the relevant question is whether the additional gain arising from using CCPS instead of PLS makes up for the cost of measuring crossbred individuals.

CCPS and PLS can be compared at the same level of resources by specifying the ratio of the costs of testing a purebred $v$. a crossbred individual and, subsequently, evaluating breeding schemes at the same total costs. In that case, testing crossbred individuals will reduce the number of purebred selection candidates, which reduces selection 
intensity and has a decreasing effect on $\Delta F$ (Figure 2). When compared at the same level of resources, therefore, changing from PLS to CCPS will give smaller increases of $\Delta F$ than the values presented in Figure 2. Methods used in this study can as well be used to balance rates of inbreeding and gain for crossbreeding schemes with a fixed total level of resources.

In this study, constraints on $\Delta F$ were achieved by increasing the number of parents. Alternatively, one can use dynamic selection algorithms that directly constrain $\Delta F$ (Meuwissen, 1997; Grundy et al., 1998). Though dynamic selection algorithms are expected to give higher $\Delta G$ at the same $\Delta F$, optimization of breeding schemes using those algorithms can only be implemented by means of computationally demanding stochastic simulation, which limits the number of alternative schemes considered in the optimization. Our methods provide good insight into the impact of different parameters and as such provide a good starting point for the optimization of breeding schemes.

\section{Acknowledgements}

This research was financially supported by the Netherlands Technology Foundation (STW), and was co-ordinated by the Life Sciences Foundation (SLW). J. A. W. acknowledges the Ministry of Agriculture, Fisheries and Food (United Kingdom) for financial support.

\section{References}

Baumung, R., Sölkner, J. and Essl, A. 1997. Selection response according to the use of pure- and/or crossbred information in crossbred animals with varying levels of dominance and additive by additive effects. Book of abstracts of the 48th annual meeting of the European Association for Animal Production, August 25-28, Vienna, Austria.

Bijma, P. and Arendonk, J. A. M. van. 1998. Maximizing genetic gain for the sire line of a crossbreeding scheme utilizing both purebred and crossbred information. Animal Science 66: 529-542.

Bijma, P. and Woolliams, J. A. 2000. Prediction of rates of inbreeding in populations selected on Best Linear Unbiased Prediction of breeding value. Genetics 156: 361-373.

Bulmer, M. G. 1971. The effect of selection on genetic variability. The American Naturalist 105: 201-211.
Grundy, B., Villanueva, B. and Woolliams, J. A. 1998. Dynamic selection procedures for constrained inbreeding and their consequences for pedigree development. Genetical Research, Cambridge 72: 159-168.

Hill, W. G. 1998. Inferences from evolutionary biology to livestock breeding. In Proceedings of the sixth world congress on genetics applied to livestock production, January 11-16, Armidale, Australia.

Jiang, X. and Groen, A. F. 1999. Combined crossbred and purebred selection for reproduction traits in a broiler dam line. Journal of Animal Breeding and Genetics 116: 111-125.

Meuwissen, T. H. E. 1991. Reduction of selection differentials in finite populations with a nested full-half-sib family structure. Biometrics 47: 195-203.

Meuwissen, T. H. E. 1997. Maximizing the response of selection with a predefined rate of inbreeding. Journal of Animal Science 75: 934-940.

Spilke, J., Groeneveld, E. and Mielenz, N. 1998. Joint purebred and crossbred (co)variance component estimation with a pseudo multiple trait model: loss in efficiency. Journal of Animal Breeding and Genetics 115: 341-350.

Turelli, M. and Barton, N. H. 1994. Genetic and statistical analyses of strong selection on polygenic traits: what, me normal? Genetics 138: 913-941.

Uimari, P. and Gibson, J. P. 1998. The value of crossbreeding information in selection of poultry under a dominance model. Animal Science 66: 519-528.

Wei, M. and Werf, J. H. J. van der. 1993. Animal model estimation of additive and dominance variances in egg production traits of poultry. Journal of Animal Science 71: 57-65.

Wei, M. and Werf, J. H. J. van der. 1994. Maximizing genetic response in crossbreds using both purebred and crossbred information. Animal Production 59: 401-413.

Woolliams, J. A. and Bijma, P. 2000. Predicting rates of inbreeding in populations undergoing selection. Genetics 154: $1851-1864$.

Woolliams, J. A., Bijma, P. and Villanueva, B. 1999. Expected genetic contributions and their impact on gene flow and genetic gain. Genetics 153: 1009-1020.

Wray, N. R. and Hill, W. G. 1989. Asymptotic rates of response from index selection. Animal Production 49: 217-227.

Wright, S. 1969. Evolution and the genetics of populations, volume 2. The theory of gene frequencies. University of Chicago, Chicago.

(Received 6 April 2000-Accepted 17 August 2000) 